\title{
The CMS Level-1 Trigger for LHC Run II
}

\author{
A. Tapper* on behalf of the CMS Collaboration \\ Imperial College London \\ E-mail: a.tappereimperial.ac.uk
}

During LHC Run II the centre-of-mass energy of pp collisions has increased up to $13 \mathrm{TeV}$ and the instantaneous luminosity has progressed towards $2 \times 10^{34} \mathrm{~cm}^{-2} \mathrm{~s}^{-1}$. In order to guarantee a successful and ambitious physics programme under these conditions, the CMS trigger system system has been upgraded. The upgraded CMS Level-1 trigger is designed to improve performance at high luminosity and large number of simultaneous inelastic collisions per crossing. The trigger design, implementation and commissioning are summarised and early performance results are described.

38th International Conference on High Energy Physics 3-10 August 2016

Chicago, USA

${ }^{*}$ Speaker. 


\section{Introduction}

At the LHC, production cross sections for the most interesting processes are many orders of magnitude lower than that of the total pp cross section. Since the ATLAS and CMS experiments cannot store all data generated in pp collisions, the experiments must select interesting events online. This is the function of the trigger systems of the experiments. In the CMS experiment this is implemented as a two-level system, with the first level (Level-1) based on custom hardware and with a software-based second level running on a large farm of computers.

With the start of LHC Run II, trigger rates have increased due to the increase in luminosity, the increase in centre-of-mass energy, and by the higher pile-up (the number of interactions per bunch crossing). The CMS detector electronics limit the Level-1 (L1) trigger rate to $100 \mathrm{kHz}$. The CMS physics programme requires the sensitivity for electroweak scale physics and for $\mathrm{TeV}$ scale searches be maintained at the level it was in Run I, which motivated the upgrade to the CMS Level-1 trigger system, described in this paper.

\section{Design, implementation and commissioning}

The legacy CMS Level-1 trigger is described in detail elsewhere [1,2]. The overall aim of the upgrade was to implement a flexible, maintainable system, capable of being adapted to the evolving CMS physics programme. The key conceptual changes planned to the design of the Level-1 trigger may be summarised as [3]:

- Muon system - the redundancy of the three muon detector systems is used early to make a high resolution muon trigger;

- Calorimeter system - boundaries have been removed by streaming data from single event into one FPGA;

- Global trigger - expandable to many more possible conditions and more sophisticated quantities, to give a richer menu of possible trigger conditions.

The key technology changes may be summarised as:

- legacy VME-based electronics replaced with microTCA, modern telecoms standard;

- system wide use of latest generation of FPGAs, Xilinx Virtex 7, in a reduced number of common platforms;

- parallel copper links replaced with serial optical links, so link speeds increased from $1 \mathrm{~Gb} / \mathrm{s}$ to $10 \mathrm{~Gb} / \mathrm{s}$;

- large optical patch panels with single fibres replaced with custom-made commercial solution (Molex Flexplane);

- online software rewritten to have more common code, modern libraries, more easily maintained. 
In order to reduce the potential risk to CMS data taking the upgrade was commissioned in parallel with the legacy trigger data taking in 2015. Trigger inputs from the calorimeters were duplicated (in FPGAs and optically) and muon trigger inputs were duplicated (endcap) and a slice commissioned (barrel). The new system was run parasitically with CMS data taking, recording results but not issuing triggers towards the end of 2015 .

\section{Trigger performance}

\subsection{Muon track finders}

Muon track finding was segmented into Barrel $(|\eta|<0.83)$, Overlap $(0.83<|\eta|<1.24)$ and Endcap $(|\eta|>1.24)$ regional processors. The motivation being the differences in background rate and magnetic field uniformity as $|\eta|$ increases. The overlap region was treated separately because it is an area where all three muon detectors contribute, and therefore more complicated than the other areas.

In contrast to the legacy muon trigger the upgrade brings together data from the three complementary detector technologies early in the track finding procedure to improve resolution and improve robustness in the case of dead detector chambers or channels.

A detailed description of the track finding algorithms is beyond the scope of this paper (more details may be found elsewhere in these proceedings [4]). In summary, pattern-based track finding is used in the endcap and overlap regions, with a look-up-table based on an MVA for $p_{T}$ assignment in the endcap. A simpler road search extrapolation track finder is used in the barrel. The global muon trigger takes muon tracks from the regional finders, sorts by muon $p_{T}$ and quality and cancels any duplicate tracks found. There is also provision for input from the calorimeter trigger to apply calorimeter energy isolation to muon candidates.

Figure 1 shows the muon trigger efficiency for a single muon trigger with $\mathrm{p}_{T}$ thresholds of 18 , 22 and $25 \mathrm{GeV}$ as a function of the offline muon $\mathrm{p}_{T}$ and $\eta$ [5]. The tag-and-probe method was used to measure the efficiency with a dataset of $Z \rightarrow \mu \mu$ events. The thresholds are typical of those used to collect data in the first half of 2016 and shows the muon trigger has good performance in $\mathrm{p}_{T}$ and across $\eta$.

\section{$3.2 \mathrm{e} / \gamma$ finder}

The upgraded e/ $\gamma$ finding algorithm [6,7] brings a number of improvements over the legacy algorithm. In particular the new algorithm is based on dynamic clustering, allowing the dimensions of the energy clustered to form the e/ $\gamma$ candidate to adapt to the pattern of energy deposited in the electromagnetic calorimeter. This gives improved energy containment for showering electrons and photo conversions without being effected significantly by pile-up. Clusters produced using this algorithm have better energy resolution than those formed by the legacy algorithm.

In addtion the shape of the clusters may be used, along with the fraction of the total energy measured as electromagnetic, to discriminate between real e/ $\gamma$ canidates and background from hadronic jets. The clusters also provide an energy-weighted position measurement which may be used to correlate objects, for example in invariant mass calculatons. 

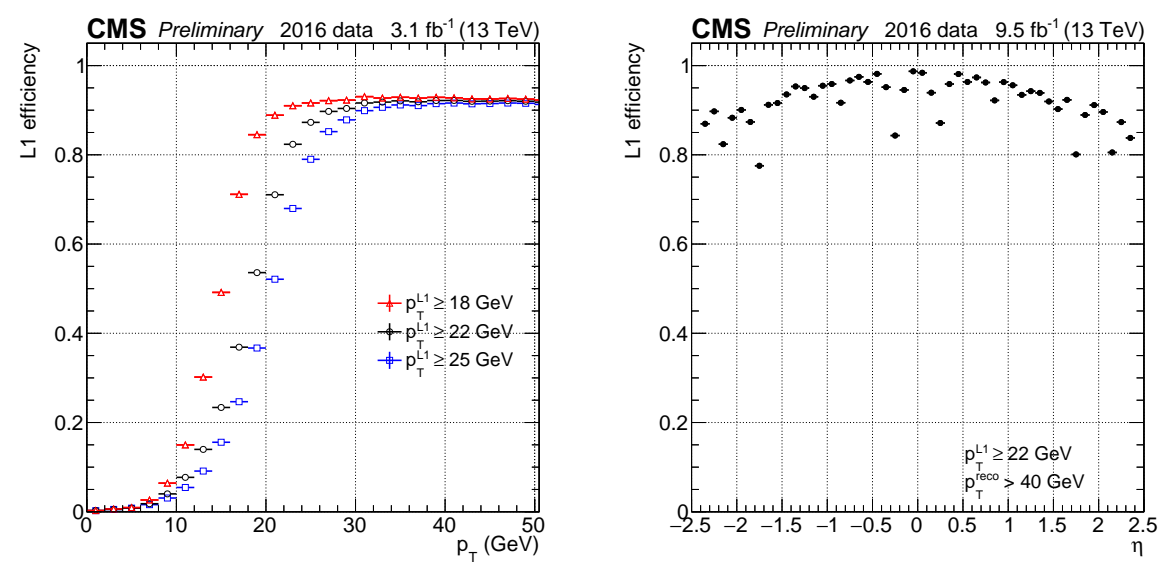

Figure 1: Muon trigger efficiency for a single muon trigger as a function of the offline muon $\mathrm{p}_{T}$ (left) and $\eta$ (right).

Isolation annuli (removing the footprint of the e/ $\gamma$ candidate) are constructed around each candidate cluster and are used to implement an isolation energy requirement, as a function of cluster $E_{T}$ and the pile-up estimated in each event.

Figure 2 (left) shows the measured trigger efficiency for a single e/ $\gamma$ with $\mathrm{E}_{T}>40 \mathrm{GeV}$ as a function of the offline $\mathrm{E}_{T}$, separately for the barrel and endcap regions of the detector [8]. The efficiency was measured using the tag-and-probe technique on a sample of $Z \rightarrow e e$ events and shows good efficiency with a sharp response, due to the excellent energy resolution.
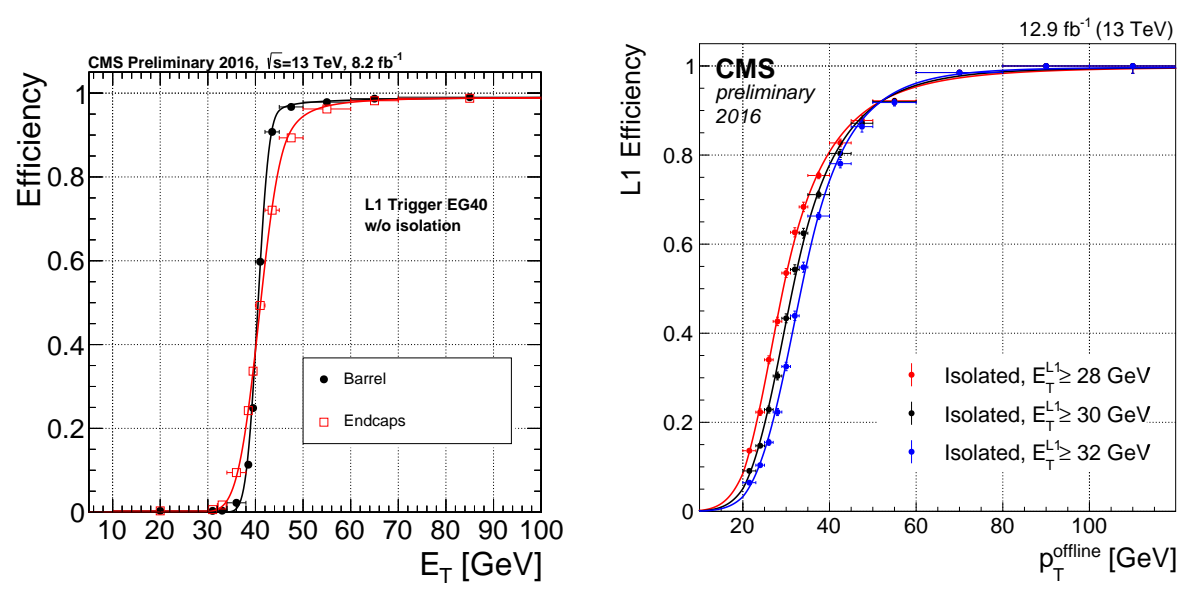

Figure 2: Trigger efficiency for single $e / \gamma$ (left) and isolated $\tau$ (right) triggers as a function of offline $\mathrm{E}_{T}$.

\section{$3.3 \tau$ finder}

The upgraded $\tau$-lepton finding algorithm $[6,7]$ is based on the same dynamic clustering as the $e / \gamma$ finder described above, optimised for $\tau$ leptons. In addtion neighbouring clusters may be 
merged to recover multi-prong $\tau$ decays. An isolation requirement may also be placed on the $\tau$ candidates, which includes whether two clusters were merged as input.

Figure 2 (right) shows the trigger efficiency for a single isolated $\tau$ with $\mathrm{E}_{T}>28,30$ and 32 $\mathrm{GeV}$, which are representative thresholds used in di- $\tau$ triggers for 2016, as a function of the offline $\mathrm{E}_{T}$ [8]. The efficiency was measured using the tag-and-probe technique on a sample of $Z \rightarrow \tau \tau$ events, where one $\tau$ decays hadronically and one to a muon, and shows good efficiency with a sharp response, due to the excellent energy resolution.

\subsection{Jet finder and energy sums}

The jet finding algorithm $[6,7]$ benefits from the increased spatial granularity of inputs compared to the legacy trigger. The algorithm is a sliding window based sum, with dimensions matched to the $\mathrm{R}=0.4$ jet finding used offline by CMS. A jet is formed by searching for a seed energy deposit above a chosen threshold and summing the energy deposits around the seed. A veto mask is applied to remove duplicate jets and areas outside the size of typical jets are used to estimate and subtract the energy within the jet from pile-up. Jet energies are calibrated as a function of jet $\mathrm{E}_{T}$ and $\eta$.

Figure 3 shows a comparison of the Level-1 trigger jet $\mathrm{E}_{t}$ with the offline jet $\mathrm{E}_{T}$ produced using a data sample triggered with an independent, single muon, trigger [8]. Level-1 and offline jets are matched geometrically and their energies compared. The result shows the Level-1 jets are well calibrated and insensitive to the effects of pile-up in the range available with this data sample. The efficiencies for single jet trigger with a range of thresholds are also shown. The results show sharp efficiency turn-on with a well calibrated $\mathrm{E}_{T}$ scale.
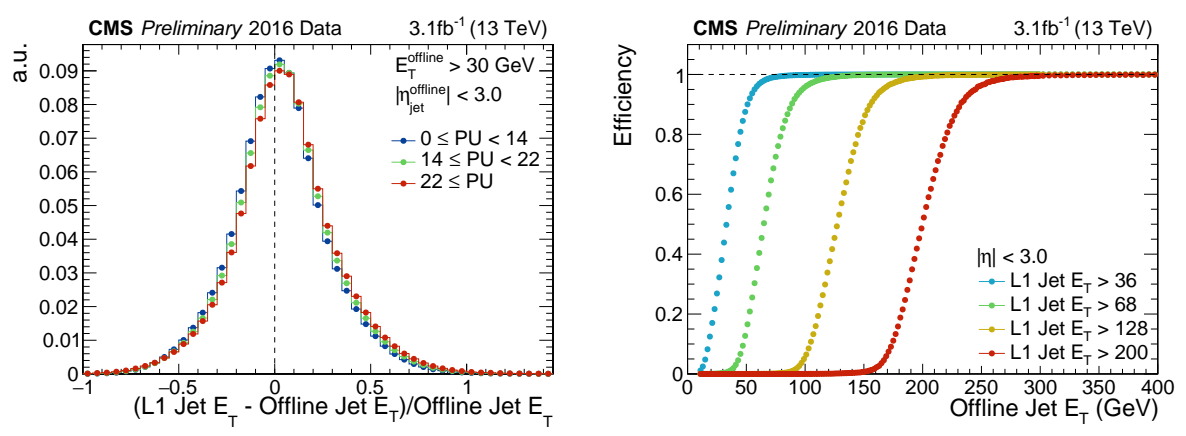

Figure 3: Comparison of Level-1 and offline jet $\mathrm{E}_{T}$ (left) and efficiencies for a range of single jet trigger thresholds, as a function of matched offline jet $\mathrm{E}_{T}$ (right).

The Level-1 jets are used to calculate the scalar sum of all jets with $\mathrm{E}_{T}>30 \mathrm{GeV}$ and $|\eta|<3$, the quantity denoted as $\mathrm{H}_{T}$ which is widely used, for example in searches for supersymmetry. The trigger efficiency for this quantity is shown in Fig. 4, as a function of the equivalent offline quantity, evaluated using a data sample triggered with an independent, single muon, trigger.

Figure 4 also shows the efficiency for the missing transverse momentum trigger, calculated as the vector sum of all energy deposits with $|\eta|<3$ and evaluated using the same sample as the $\mathrm{H}_{T}$ [8]. This quantity is widely used in searches for physics beyond the Standard Model. 

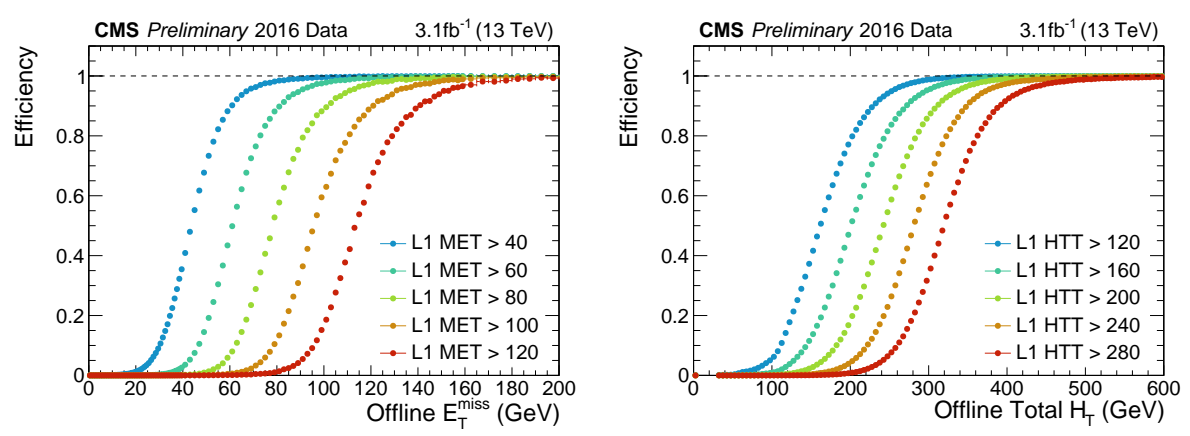

Figure 4: Efficiencies for the $\mathrm{H}_{T}$ (left) and missing transverse momentum (right) triggers.

\section{Summary}

Run II at the LHC is a very challenging environment to search for new physics and measure the properties of the Higgs boson. The newly installed CMS Level-1 trigger upgrade tackles these challenges and has run reliably, providing CMS with high-quaity physics data since the start of the 2016 run. The system is based on state-of-the-art, FPGA based, very high bandwidth processors with sophisticated, programmable algorithms. Early performance results from the 2016 run have been documented and demonstrate good performance. The design of the trigger allows flexibility to evolve with the requirements of the CMS physics programme and will also provide lessons for the design of the trigger upgrade planned for high luminosity LHC running in around a decade from now.

\section{References}

[1] CMS Collaboration, "The TriDAS Project Technical Design Report, Volume 1: The Trigger Systems", CMS-TDR-006, CERN-LHCC-2000-038 (2000). https://cds.cern.ch/record/706847

[2] CMS Collaboration, "The CMS trigger system", submitted to JINST. arXiv:1609.02366

[3] CMS Collaboration, "CMS Technical Design Report for the Level-1 Trigger Upgrade", CMS-TDR-012, CERN-LHCC-2013-011 (2013). https://cds.cern.ch/record/1556311

[4] D. Rabady et al., "Upgrade of the CMS muon trigger system in the barrel region", in these proceedings.

[5] CMS Collaboration, "L1 muon trigger performance - ICHEP16 dataset", CMS-DP 2016-050 (2016). https://cds.cern.ch/record/2202986

[6] A. Zabi et al., "Triggering on electrons, jets and tau leptons with the CMS upgraded calorimeter trigger for the LHC RUN II", JINST 11 (2016) 02, C02008.

[7] B. Kreis et al., "Run 2 Upgrades to the CMS Level-1 Calorimeter Trigger", JINST 11 (2016) C01051.

[8] CMS Collaboration, "CMS L1 Calorimeter Trigger performance in 2016 data”, CMS-DP 2016-044 (2016). https://cds.cern.ch/record/2202966 